\title{
Just Another Acute Chest Pain? Case of Contained Rupture of a Saphenous Venous Graft Pseudo-Aneurysm Mimicking Acute Coronary Syndrome
}

\author{
Ross Biggs ${ }^{\mathrm{a}, \mathrm{c}}$, Mahek Shah ${ }^{\mathrm{b}}$, Matthew Martinez ${ }^{\mathrm{b}}$, Raman Dusaj ${ }^{\mathrm{b}}$, \\ Larry Jacobs ${ }^{b}$
}

\begin{abstract}
Rupture of a saphenous vein graft (SVG) pseudo-aneurysm presenting as a non-ST elevation myocardial infarction is a rarely reported, but serious complication of prior coronary artery bypass graft surgery. We present a case of a 76-year-old man who presented with severe chest pain and elevated cardiac biomarkers, and was found to have a contained pseudo-aneurysm rupture at the site of anastomosis of a 15 -year SVG with the ascending aorta requiring surgical repair. We also review presenting symptoms, the current literature and treatment approaches for SVG pseudo-aneurysm.
\end{abstract}

Keywords: Pseudo-aneurysm rupture; Acute coronary syndrome; Chest pain

\section{Introduction}

Rupture of a saphenous vein graft (SVG) pseudo-aneurysm is a rare but potentially life-threatening complication in some patients who underwent coronary artery bypass graft $(\mathrm{CABG})$ surgery. Formation of aneurysmal dilation of aortocoronary SVGs can be early or late after a bypass surgery. It is likely that the underlying pathophysiology is multifactorial as a combination of several proposed theories. Early aneurysm formation may be related to weakness of the venous wall, disruption of sutures at the anastomosis and surgical technical factors, among others [1-3]. Late aneurysms have been reported several years after surgery and their development may be linked to underlying atherosclerotic graft degeneration, subsequent weakening of the smooth muscle wall, vessel wall ischemia,

Manuscript accepted for publication December 13, 2016

aDivision of Internal Medicine, Lehigh Valley Hospital Network, Allentown, PA, USA

bDivision of Cardiology, Lehigh Valley Hospital Network, Allentown, PA, USA

${ }^{\mathrm{c} C o r r e s p o n d i n g ~ A u t h o r: ~ R o s s ~ B i g g s, ~ D e p a r t m e n t ~ o f ~ I n t e r n a l ~ M e d i c i n e, ~ L e h i g h ~}$ Valley Health Network, 1255 S Cedar Crest Blvd, Suite 300, Allentown, PA 18103, USA. Email: Ross.Biggs@1vhn.org

doi: https://doi.org/10.14740/jmc2705w and the long-term impact of arterial circulation and exposure to a high pressure pulsatile system on the venous graft and anastomosis with the aorta [4-6].

Prior reported cases show that SVG pseudo-aneurysms are highly variable in their clinical presentation, with a third of the patients being asymptomatic at time of presentation and half of the reported cases reporting chest pain [7]. Incidence of pseudo-aneurysm after CABG has been reported to be < $1 \%$ in one case series, and rupture occurs in about $11 \%$ of the reported cases [7, 8]. We report a case of a 76-year-old man presenting with acute onset chest pain and elevated biomarker levels, who was found to have a ruptured SVG pseudo-aneurysm at its junction with ascending aorta, 15 years after initial CABG surgery.

\section{Case Report}

A 76-year-old male presented to the emergency department with 2 days of pleuritic left-sided chest pain. He described the pain as severe, sharp, radiating to his neck, worse with activity or deep inspiration and partially relieved with sublingual nitroglycerine. The patient had undergone triple-vessel CABG 15 years ago with SVGs to the second obtuse marginal $\left(\mathrm{OM}_{2}\right)$ and right coronary artery (RCA), and a left internal mammary artery (LIMA) graft to the left anterior descending artery (LAD). Previous cardiac catheterization had revealed complete occlusion of the SVG to RCA, a patent LIMA graft to the LAD and $95 \%$ stenosis at the ostium of the SVG to $\mathrm{OM}_{2}$, subsequently revascularized using a drug-eluting stent.

Initial workup included an electrocardiogram (ECG) that demonstrated inferior Q-waves without evidence of acute ischemic changes. Laboratory tests were unremarkable apart from an elevated troponin-I of $2.16 \mathrm{ng} / \mathrm{mL}$. A computed tomography (CT) scan of the chest with contrast showed a 2.3 $\times 3.4 \mathrm{~cm}$ lobulated pseudo-aneurysm of the anterior ascending aorta with evidence of local extravasation of dye beyond the pseudo-aneurysm and surrounding hematoma (Fig. 1a). A bedside transthoracic echocardiogram was emergently performed which showed no evidence of pericardial effusion.

The patient was taken for emergent catheterization and aortography, which confirmed a pseudo-aneurysm of the anterior ascending aortic wall right at the origin of the SVG to RCA but no active extravasation of dye (Fig. 1b, Supplemen- 

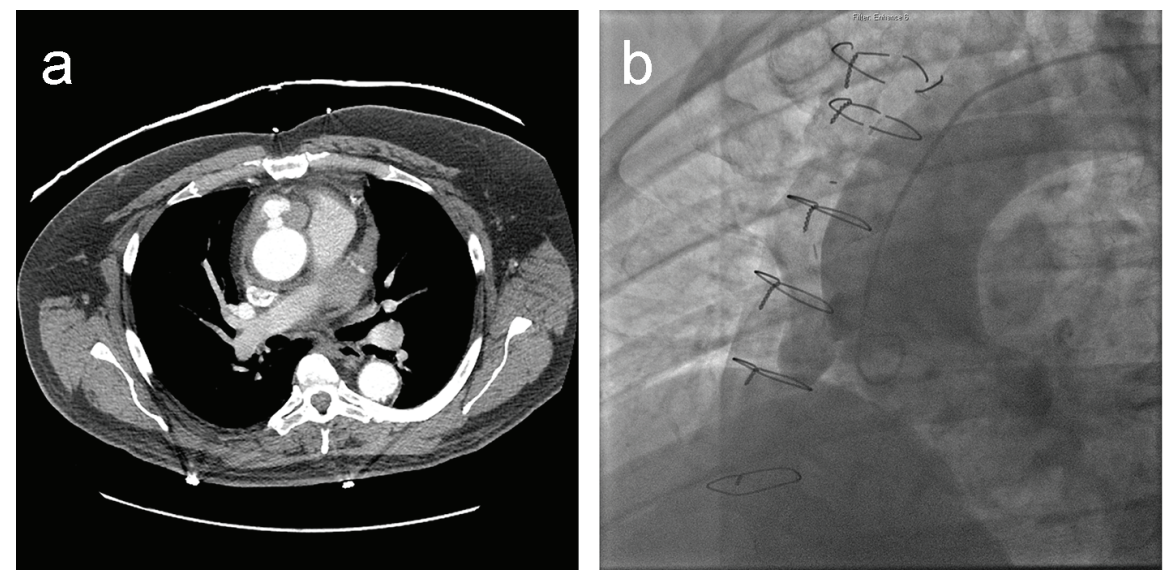

Figure 1. (a) Cross-sectional CT image of the chest revealing a $2.3 \times 3.4 \mathrm{~cm}$ lobulated pseudo-aneurysm of the anterior ascending thoracic aorta, with moderate surrounding hematoma and a small degree of hemopericardium. (b) LAO aortography demonstrating a pseudo-aneurysm of the SVG anastamosis to the RCA.

tary video 1, www.journalmc.org). Due to persistent chest discomfort, the patient was taken for emergent re-do sternotomy. Intra-operatively, a transesophageal echocardiogram revealed a thickened proximal ascending aorta with pseudo-aneurysmal changes and possible thrombus formation within. After initiating peripheral bypass, decompression of the heart and crossclamping the aorta, a large hematoma was noted at the site of pseudo-aneurysm confirming contained rupture of the underlying pseudo-aneurysm. The pseudo-aneurysm was unroofed, vein graft to the aorta was resected and a bovine pericardial patch was placed to successfully repair the pseudo-aneurysm.

Postoperatively, the patient was weaned off pressors within $48 \mathrm{~h}$. The patient had a prolonged hospital stay with development of ileus and significant deconditioning eventually being discharged home 12 days postoperatively. On outpatient follow-up, patient was seen to have recovered well and a repeat CT scan revealed no abnormalities of the ascending aorta.

\section{Discussion}

SVG pseudo-aneurysm formation has been reported in the literature with an incidence as low as $0.07 \%$ in one case series [9]. However, considering the fact that a significant proportion of patients are asymptomatic and there are currently no guidelines on how to screen patients at risk, prior estimates are an underestimation. In a 2015 systematic review of SVG pseudo-aneurysms, Smer et al reported that $48.6 \%$ cases presented with chest pain, followed by an asymptomatic presentation in $30.5 \%$ of the patients. A majority of the reported symptomatic cases were male, presenting between 11 and 20 years after CABG [7]. Other extreme presentations are present in literature, with pseudo-aneurysms presenting as superior vena cava syndrome secondary to direct compression [10] or even large lung masses [11].

Diagnosis of a new SVG pseudo-aneurysm can be challenging and a combination of imaging modalities may be required to adequately characterize the pseudo-aneurysm, its extent and surrounding structures. In a systematic review by
Ramirez et al [1], the authors reported that $66 \%$ of the cases had cardiac catheterization, $60 \%$ received $\mathrm{CT}$ imaging (with or without contrast) and magnetic resonance imaging was used in $13 \%$. Chest X-ray was performed in over 54\% cases; however, their use in diagnosis may be quite limited. Use of transthoracic or transesophageal echocardiogram was noted in $28 \%$ of the reviewed cases. In our patient, multiple imaging modalities (chest X-ray, CT chest, echocardiogram and cardiac catheterization) were performed to characterize the pseudo-aneurysm and these modalities complemented each other and assisted in determining the best approach to treatment.

Several treatment modalities have been attempted in repairing pseudo-aneurysms with an individualized patient-dependent approach which steered the selection of one modality over another. Open surgical exploration with re-do sternotomy is the most common therapy of choice due to its definitive treatment with direct repair of the pseudo-aneurysm, but coil embolization [12], covered stenting [13], thrombin injection, Amplatzer device placement and conservative management [14] have all been used in the management of SVG pseudoaneurysms. To date, literature on SVG pseudo-aneurysm is limited to case reports and case series, hence the treatment modality chosen is dependent on physician/facility experience and the clinical factors in each case. Head to head comparison between the different modalities has not been performed. In our case, due to ongoing symptoms and CT findings suggestive of a contained pseudo-aneurysmal rupture, a surgical approach was implemented successfully.

In a paper by Sareyyupoglu et al, a retrospective review of 16 patients was performed with median follow-up of 7 years. They reported that survival was $83 \%$ at 5 years and $72 \%$ at 10 years after SVG aneurysm repair [15]. Due to these findings, they proposed surgical exploration for symptomatic patients, aneurysms larger than $1 \mathrm{~cm}$ in diameter, diminished graft flow or presence of structural complications (fistula, compression or rupture). On the other hand, other studies have reported positive outcomes with percutaneous techniques or even a conservative strategy in some cases $[5,9]$.

Ramirez et al reported a relatively high in-hospital and/ 
or 30 -day mortality rate in their systematic review at $13.9 \%$, despite the presence of several asymptomatic and incidentally discovered cases [1]. They noted that both percutaneous and surgical approaches were beneficial, with the former having a greater role in patients at high risk for re-do surgery, feasibility of procedure and the absence of viable myocardium in the territory supplied by the graft. They found that the patients treated percutaneously had the lowest mortality followed by surgery and conservative management. A third of the cases with SVG aneurysms measuring $<20 \mathrm{~mm}$ had adverse outcomes, making size of the aneurysm an unreliable prognostic marker. Due to the rarity and complexity of this postoperative complication, large prospective or randomized control trials would be difficult to perform. Until then, the role of screening for, and monitoring small pseudo-aneurysms remains unclear.

\section{Conclusion}

In conclusion, our case stresses the importance of having a wide differential diagnosis in patients presenting with acute chest pain, especially in the presence of prior CABG. We recommend that awareness of this complication should be present among the caretakers, and should be strongly considered in suspicious cases as it may represent a high-risk population. A multimodality imaging approach should be implemented promptly with the goal to rule out mechanical complications and decision for treatment can be made on a case to case basis depending on patient/physician preference and experience.

\section{Conflicts of Interest}

None.

\section{References}

1. Ramirez FD, Hibbert B, Simard T, Pourdjabbar A, Wilson KR, Hibbert R, Kazmi M, et al. Natural history and management of aortocoronary saphenous vein graft aneurysms: a systematic review of published cases. Circulation. 2012;126(18):2248-2256.

2. Mohara J, Konishi H, Kato M, Misawa Y, Kamisawa O, Fuse K. Saphenous vein graft pseudoaneurysm rupture after coronary artery bypass grafting. Ann Thorac Surg. 1998;65(3):831-832.

3. de Haan HP, Huysmans HA, Weeda HW, Bosker HA, Buis
B. Anastomotic pseudoaneurysm after aorto-coronary bypass grafting. Thorac Cardiovasc Surg. 1985;33(1):5556.

4. Kallis P, Keogh BE, Davies MJ. Pseudoaneurysm of aortocoronary vein graft secondary to late venous rupture: case report and literature review. Br Heart J. 1993;70(2):189192.

5. Memon AQ, Huang RI, Marcus F, Xavier L, Alpert J. Saphenous vein graft aneurysm: case report and review. Cardiol Rev. 2003;11(1):26-34.

6. McGeachie JK, Prendergast FJ, Morris PJ. Vein grafts for arterial repair: an experimental study of the histological development of the intima. Ann R Coll Surg Engl. 1983;65(2):85-89.

7. Smer A, Alla V, Chandraprakasam S, Abuzaid A, Saurav A, Holmberg J. Saphenous venous graft pseudoaneurysm: a review of the literature. J Card Surg. 2015;30(1):70-73.

8. Keon WJ, Bedard P, Akyurekli Y, Brais M. Causes of death in aortocoronary bypass surgery: experience with 1,000 patients. Ann Thorac Surg. 1977;23(4):357-360.

9. Dieter RS, Patel AK, Yandow D, Pacanowski JP, Jr., Bhattacharya A, Gimelli G, Kosolcharoen P, et al. Conservative vs. invasive treatment of aortocoronary saphenous vein graft aneurysms: Treatment algorithm based upon a large series. Cardiovasc Surg. 2003;11(6):507-513.

10. Vargas-Estrada A, Edwards D, Bashir M, Rossen J, Zahr F. Giant saphenous vein graft pseudoaneurysm to right posterior descending artery presenting with superior vena cava syndrome. World J Cardiol. 2015;7(6):351-356.

11. Abbasi M, Soltani G, Shomali A, Javan H. A large saphenous vein graft aneurysm one year after coronary artery bypass graft surgery presenting as a left lung mass. Interact Cardiovasc Thorac Surg. 2009;8(6):691-693.

12. Maybrook RJ, Sharma S, Gupta K, Wiley M, Parashara D. Percutaneous closure of a coronary artery-to-vein graft anastomotic pseudoaneurysm presenting as acute coronary syndrome after recent coronary artery bypass grafting. Tex Heart Inst J. 2015;42(3):265-269.

13. Johnson NP, Dasgupta A, Appel D, Beohar N. Post-intervention coronary pseudoaneurysm treated with a covered stent. Tex Heart Inst J. 2012;39(3):448-449.

14. Hamid T, Malik N. Saphenous vein graft pseudoaneurysm: a case for medical management. J Coll Physicians Surg Pak. 2014;24(Suppl 3):S169-170.

15. Sareyyupoglu B, Schaff HV, Ucar I, Sundt TM, 3rd, Dearani JA, Park SJ. Surgical treatment of saphenous vein graft aneurysms after coronary artery revascularization. Ann Thorac Surg. 2009;88(6):1801-1805. 